\title{
Osteogénesis imperfecta: estudio de la calidad de vida en los niños
}

\author{
Osteogenesis imperfecta: quality of life in children
}

\author{
Dra. Virginia Fano ${ }^{a}$, Dra. Mariana del Pino ${ }^{a}$, Dra. Mercedes Rodríguez Celin ${ }^{a}$, \\ Lic. Susana Bucetab y Dra. M. Gabriela Obregón
}

\section{RESUMEN}

La calidad de vida relacionada con la salud (CVRS) en los niños con osteogénesis imperfecta (OI) ha sido poco comunicada. Se la evaluó con el cuestionario Peds QL versión 4.0, en 65 familias y 42 niños, con una media de edad de 7,76 años ( 35 con OI de tipo I y 30 con OI de tipo III-IV). Hubo diferencias significativas en el dominio físico según los niños y los padres, entre los niños con OI de tipo I y aquellos con OI de tipo III-IV. En el dominio social solo los padres consideraron menor CVRS en las formas III-IV.

En el análisis multivariado de las formas graves, para los padres la mejor CVRS se asoció con menor déficit de estatura (coef. $\beta=3,8 ; p=0,039$ ), menor número de fracturas (coef. $\beta=0,69$; $p=0,003$ ) y mayor dosis de pamidronato (coef. $\beta=1,44 ; p=0,037)$. Para los niños, la CVRS fue mejor cuando hubo adherencia al tratamiento (coef. $\beta=19,41 ; p=0,03$ ).

Palabras clave: osteogénesis imperfecta, calidad de vida relacionada con la salud, CVRS, Peds QL 4.0.

Grupo Interdisciplinario para la atención de pacientes con osteogénesis imperfecta.

a. Servicio de Crecimiento y

Desarrollo.

b. Servicio de Kinesiología. c. Servicio de Genética. Hospital Nacional de Pediatría "Prof. Dr. Juan P. Garrahan", Buenos Aires, Argentina.

\section{Correspondencia:} Dra. Virginia Fano: virginiafano@gmail. com

Conflicto de intereses: Ninguno que declarar.

Recibido: 28-1-2013 Aceptado: 10-5-2013

\section{ABSTRACT}

The impact produced by osteogenesis imperfecta in childrens' quality of life almost has not been reported; 65 children, 38 males, were evaluated according to the questionnaire PedsQL 4.0 Argentinean Spanish version. Median age was 7.76 years; 35 children with OI type I were compared with 30 type III-IV (according to Sillence Classification) finding significant difference in the physical domain in both visions, children and parents, and only in parents' vision in the social area. Multivariate analysis showed an association between better PedQL scores and treatment compliance (coef. $\beta=19.41 p=0.03$ ) in children's vision. In parental report on the other hand, the association was found with greater pamidronate doses (coef. $\beta 1.44 \mathrm{p}=0.037$ ), lower height compromise (coef. $\beta=3.8 ; p=0.039$ ) and less number of fractures (coef. $\beta=0.69 ; p=0.003$ ).

Key words: Health-related quality oflife, osteogenesis imperfecta, Peds Ql 4.0.

http:/ /dx.doi.org/10.5546/aap.2013.328

\section{INTRODUCCIÓN}

La osteogénesis imperfecta (OI) es una enfermedad hereditaria del tejido conectivo con una prevalencia de 1 en 15000 RN. ${ }^{1}$ Los signos clínicos son fragilidad ósea, compromiso variable de la estatura y deformaciones esqueléticas. También puede haber alteración dentaria, escleróticas azules, hiperlaxitud articular y sordera, ya que tiene una variabilidad clínica muy amplia. $^{2}$

En las últimas décadas, se han producido cambios en la atención de los niños con OI; las estrategias terapéuticas actuales comprenden actividad física, cirugías ortopédicas, y administración de fármacos antirreabsortivos y analgésicos, como el pamidronato de uso intravenoso., Para ello, se realiza una intervención terapéutica personalizada a fin de maximizar las capacidades y la integración social.

Los indicadores clásicos de salud, como la tasa de mortalidad o el número de internaciones, resultan insuficientes para evaluar el impacto de la enfermedad y de los tratamientos en la vida de los pacientes. ${ }^{5}$

Existen indicadores que permiten una evaluación más integral de la salud de los niños, como las escalas de estado funcional y bienestar, y las que miden la calidad de vida relacionada con la salud (CVRS). La CVRS es una construcción multidimensional que intenta medir el impacto de la enfermedad en la calidad de vida tomando en consideración la perspectiva del paciente. En Sudamérica, la información sobre la CVRS en los pacientes con OI es escasa. ${ }^{6-11}$

El objetivo del presente trabajo fue evaluar la CVRS utilizando el cuestionario PedsQL 4.0 (American Pediatric Quality of Life Inventory) en un grupo de pacientes con OI. 


\section{PACIENTES Y MÉTODOS}

Estudio analítico transversal y observacional.

Se incluyeron todos los pacientes que consultaron al equipo de atención multidisciplinaria de niños con OI del Hospital de Pediatría "Prof. Dr. Juan P. Garrahan" en el período de julio a diciembre de 2007. Los criterios de inclusión fueron: pacientes con diagnóstico de OI, confirmado por clínica, radiología y consulta genética en edades comprendidas entre 2 y 18 años. Se utilizó la clasificación de Sillence, que divide la enfermedad en tipo I: leve compromiso de la estatura, fragilidad ósea moderada; tipo II: letal, tipo III: facies triangular, compromiso grave de la estatura y progresivamente deformante, y tipo IV: forma intermedia. ${ }^{11}$ Se excluyeron los niños que presentaban otra enfermedad crónica y otras formas muy infrecuentes de OI. El proyecto fue aprobado por la Dirección de Docencia e Investigación y por el Comité de Ética del hospital. El consentimiento informado fue firmado por el adulto a cargo y se solicitó a todos los niños de 13 a 18 años un asentimiento.

La evaluación de la calidad de vida se realizó con el cuestionario PedsQl 4.0 en su versión validada española-argentina. Este cuestionario consta de 23 ítems, divididos en 4 dominios que comprenden los aspectos físico, emocional, social y escolar, con un rango de aplicación entre 2 y 18 años de edad, y cuyos resultados se expresan con puntajes entre 0 y 100; a mayor puntaje, mejor CVRS.

Si bien no existen valores normales o aceptables, se tomaron como referencia los del estudio argentino de validación del PedQl, que discriminó la población sana de la crónicamente enferma, con valores mayores de 70 ( 72,72 contra $66,87) .{ }^{6}$ Los cuestionarios fueron completados con anterioridad a la realización de la consulta médica administrados por un solo operador (MRC). Padres y niños completaron el cuestionario en forma independiente.

En el caso de los niños de entre 2 y 4 años, se evaluó solo la visión de los padres; los niños de entre 5 y 7 años fueron asistidos para responder según la metodología estandarizada de la toma del cuestionario.

Todos los niños fueron evaluados globalmente; los resultados de la evaluación antropométrica, el estado funcional y la terapéutica se publicaron previamente. ${ }^{12}$ Las variables sociales de las familiares evaluadas fueron: nivel de educación materna, nivel socioeconómico según tuvieran o no necesidades básicas insatisfechas (INDEC), subsidio de seguridad social, obra social y adherencia al tratamiento. Las variables clínicas de los niños fueron: sexo, edad, tipo de OI, escolaridad, tiempo promedio de seguimiento, número de fracturas, déficit de estatura, presencia o no de desejes en los miembros, tratamiento y dosis de pamidronato (indicado en las formas graves de OI y en el compromiso grave de la columna).

Métodos estadísticos: para el análisis bivariado se aplicaron las pruebas de comparación de dos grupos de observaciones independientes para datos continuos o categóricos, según correspondiera. Se consideró un valor de $\alpha=0,05$. Se realizó una regresión lineal múltiple de a pasos considerándose como variable dependiente el puntaje de calidad de vida del dominio físico de 0-100 y como variables independientes se incluyeron en el modelo aquellas que en el análisis bivariado tuvieron un valor de $p<0,25$.

\section{RESULTADOS}

Del total de 65 pacientes evaluados (38 niñas y 27 varones), 35 correspondieron al tipo I, 28 al tipo III y 2 al tipo IV. Para el análisis de los datos se agruparon los pacientes de tipo III y IV. El 65\% de los casos fueron familiares, con herencia $\mathrm{AD}$; el resto, mutación de novo.

La mediana de edad fue de 7,76 años $(r=1,89$ $18,91)$; en el tipo I de $8,78(r=2,37-17,42)$ y en el tipo III-IV de 7,59 años $(r=1,89-18,91)$, sin diferencias entre ambos grupos.

El tiempo promedio de seguimiento de los niños en el momento de la toma del cuestionario fue de 4,75 años, con un rango entre 0,00 y 14,72 años. Dos pacientes fueron evaluados en la primera consulta. El 100\% de los niños en edad estaban escolarizados (56 pacientes). La mayoría de ellos concurrían a una escuela común y 2 , a una escuela especial.

En la Tabla 1 se observan las características sociales de las familias y clínicas de los niños según el tipo de OI. Los resultados de la evaluación de la calidad de vida se observan en la Tabla 2, donde se muestran los valores según la visión de los niños $(n=42)$ o sus cuidadores $(n=65)$ y la gravedad de la condición. Se compararon los puntajes del cuestionario de CVRS en todos los dominios entre ambos grupos I contra III y IV, y se encontró una diferencia significativa en el dominio físico. En las otras áreas evaluadas no se hallaron diferencias, a excepción del área social, en la que se observó una diferencia significativa en la respuesta de los 
padres, que otorgaron menores valores de calidad de vida a las formas III y IV.

Se realizó un análisis de regresión lineal entre las variables independientes estudiadas y los puntajes de calidad de vida en el dominio físico de las formas graves. Las variables que en el análisis bivariado arrojaron un valor de $p<0,25$ fueron: para la respuesta de los niños: número de fracturas $(p=0,016)$, adherencia al tratamiento $(p=0,11)$, subsidio de discapacidad $(p=0,16)$, dosis de pamidronato $(p=0,24)$ y déficit de estatura $(p=0,0047)$. Para la respuesta de los padres fueron: número de fracturas $(p=0,02)$, subsidio de discapacidad $(p=0,17)$, dosis de pamidronato $(p=0,21)$ y déficit de estatura $(p=0,0004)$.

En el análisis de regresión lineal múltiple las variables asociadas a los mejores puntajes de calidad de vida en la visión de los niños fue la adherencia al tratamiento (coef. $\beta=19,41 ; p=0,03$ ) y en la respuesta de los adultos, mayor dosis de pamidronato (coef. $\beta=1,44 ; p=0,037$ ), menor déficit de estatura (coef. $\beta=3,8 ; p=0,039$ ) y menor número de fracturas (coef. $\beta=0,69 ; p=0,003$ ).

\section{DISCUSIÓN}

El concepto de calidad de vida relacionada con la salud se incorpora en la discusión médica como medida del bienestar de los pacientes desde hace relativamente poco tiempo.

Los profesionales que atendemos a pacientes con enfermedades poco frecuentes referimos los objetivos del seguimiento en función de la mejora del bienestar del paciente, pero este puede tener un significado diferente desde la mirada de los pacientes, sus padres y el equipo tratante, ${ }^{7}$ por lo que resultaba necesario considerar los aspectos de la CVRS. ${ }^{8}$

Se han publicado pocos trabajos sobre la calidad de vida en condiciones genéticas, solo 12 en la edad pediátrica y muy pocos en OI. ${ }^{13}$

TABLA 1. Osteogénesis imperfecta: características generales de la población

\begin{tabular}{|c|c|c|c|}
\hline \multicolumn{4}{|c|}{ Características sociales de las familias } \\
\hline & & & $n(\%)$ \\
\hline \multirow[t]{3}{*}{ Educación materna } & \multicolumn{2}{|c|}{ Primaria incompleta } & $7(10,8)$ \\
\hline & \multicolumn{2}{|c|}{ Primaria completa } & $29(44,6)$ \\
\hline & \multicolumn{2}{|c|}{ Secundario completo } & $29(44,6)$ \\
\hline \multicolumn{2}{|l|}{$\begin{array}{l}\text { Necesidades básicas } \\
\text { insatisfechas }\end{array}$} & Sí & $22(33,8)$ \\
\hline \multicolumn{2}{|c|}{ Subsidio seguridad social } & Sí & $26(40)$ \\
\hline \multicolumn{2}{|l|}{ Obra social } & Sí & $41(63)$ \\
\hline \multicolumn{2}{|c|}{ Adherencia al tratamiento } & Sí & $46(70,8)$ \\
\hline
\end{tabular}

\begin{tabular}{lcc}
\hline \multicolumn{3}{c}{ Características clínicas de los niños según el tipo de OI } \\
\hline & Tipo I $(n=35)$ & Tipo III y IV $(n=30)$ \\
\hline $\begin{array}{l}\text { Déficit de estatura Z } \\
\text { (x } \pm \text { ES mín/máx) }\end{array}$ & $-1,4 \pm 0,29$ & $-5,64 \pm 0,48$ \\
$\begin{array}{l}\text { Fracturas } \\
\text { (mediana y rango) }\end{array}$ & $-5,25 / 2,1$ & $-11,2 /-0,2$ \\
$\begin{array}{l}\text { Presencia de desejes } \\
\text { de miembros (\%) }\end{array}$ & 28,6 & $22(2-100)$ \\
$\begin{array}{l}\text { Tratamiento con } \\
\text { pamidronato (\%) }\end{array}$ & 26 & 86,7 \\
\hline
\end{tabular}

TABLA 2. Calidad de vida: puntaje promedio, desviación estándar y error estándar en la visión de los niños y sus padres

\begin{tabular}{|c|c|c|c|c|c|c|c|}
\hline \multirow{3}{*}{ Dominio } & \multicolumn{7}{|c|}{ Respuesta de los niños $n=42$} \\
\hline & \multicolumn{3}{|c|}{ Tipo I $n=25$} & \multicolumn{3}{|c|}{ Tipo III y IV $n=17$} & \multirow{2}{*}{$p$} \\
\hline & Media & EE & DE & Media & EE & DE & \\
\hline Físico & 66,75 & 4,6 & 22,9 & 48,7 & 5,3 & 21,7 & 0,014 \\
\hline Emocional & 70 & 3,8 & 18,8 & 67,35 & 3,18 & 13,12 & 0,62 \\
\hline Escolaridad & 76 & 3,4 & 17,3 & 72,35 & 3,62 & 14,91 & 0,48 \\
\hline \multirow[t]{2}{*}{ Social } & 67,2 & 4,41 & 22,10 & 66,76 & 4,81 & 19,84 & 0,89 \\
\hline & \multicolumn{7}{|c|}{ Respuesta de los padres $n=65$} \\
\hline Dominio & \multicolumn{3}{|c|}{ Tipo I $n=35$} & \multicolumn{3}{|c|}{ Tipo III y IV $n=30$} & $p$ \\
\hline Físico & 64,55 & 3,7 & 21,95 & 43,08 & 3,8 & 20,81 & 0,0002 \\
\hline Emocional & 62 & 3,5 & 20,5 & 63,66 & 3,5 & 19,3 & 0,74 \\
\hline Escolaridad & 74,8 & $3,3 n=31$ & 18,2 & 70,1 & 4,1 & $19,9 n=23$ & 0,47 \\
\hline Social & 72 & 3,74 & 22,10 & 67,75 & 3,14 & 17,21 & 0,01 \\
\hline
\end{tabular}

EE: error estándar. DE: desvío estándar. 
Nuestros resultados de CVRS muestran menores puntajes en el área física en toda la muestra y menores a mayor compromiso esquelético. Engelbert halló una relación directa entre el estado de bienestar y las habilidades motoras. ${ }^{14}$

Widmann, en un estudio en adultos, ${ }^{8}$ relacionó la calidad de vida y la funcionalidad, entendida como la capacidad de marcha independiente, que fue de $67 \%$ en las formas leves y de $17 \%$ en las formas graves, y no encontró que los bajos niveles de funcionalidad e independencia se relacionaran con un peor desempeño en las habilidades sociales ni académicas.

Van Brussell, ${ }^{15}$ observó los mejores resultados de movilidad con el aumento de la actividad física y la capacidad aeróbica. Finalmente, se ha relacionado la CVRS y el tratamiento con pamidronato con una mejor respuesta en los menores de 7 años. ${ }^{10}$ Nuestros resultados muestran una asociación entre la medicación con pamidronato y los mejores puntajes de CVRS en el análisis multivariado en la respuesta de los padres.

Los resultados de los dominios emocional, escolar y social señalan que, según la perspectiva de los niños en esta evaluación, no se observa una dificultad, si bien reconocemos las limitaciones de este estudio, como las diferentes edades de los niños evaluados, las distintas estructuras familiares y sociales, y las de la propia encuesta.

Observamos, en consonancia con otras investigaciones, que los valores de CVRS en la visión de los padres son menores y, en este estudio, el compromiso de la esfera social solo se expresó en la mirada de los padres y cuidadores.

Por último, la CVRS de nuestros pacientes es similar a la publicada en los niños con otras enfermedades crónicas, como trasplante de médula ósea, enfermedad pulmonar crónica, inmunodeficiencia adquirida, insuficiencia renal crónica y cardiopatías congénitas, en la misma institución. ${ }^{7}$

\section{CONCLUSIONES}

En los niños con OI de tipo III-IV, se encontró una disminución en la CVRS en el dominio físico, tanto para los padres como para los propios niños, y hubo una percepción de menor calidad de vida en el dominio social sólo de parte de los padres.

El menor déficit de estatura, el menor número de fracturas, el tratamiento con mayores dosis de pamidronato y la adherencia al tratamiento fueron las variables relacionadas con una mejor calidad de vida en las formas graves de la enfermedad.

\section{Agradecimientos}

A los niños y sus familias.

A la doctora Mariana Roizen, por su lectura crítica.

\section{BIBLIOGRAFÍA}

1. Kuurila K, Kaitila I, Johanson R, Grenman L. Hearing loss in finish adults with Osteogenesis Imperfecta:a nationwide survey. Ann Otol Rhin and Laryngol 2002;111 (10):939-46.

2. Spranger J. Osteogenesis Imperfecta. En: Bone Dysplasia. 3th edition. Oxford University Press 2012.

3. CastilloH,Samson-Fang L.American Academy for Cerebral Palsy and Developmental. Effect of bisphosphonates in children with Osteogenesis Imperfecta: an AACPDM systematic review. Devl Med Child Neurol 2009; 51(1):17-29.

4. Glorieux FH, Bishop NJ, Plotkin H, Chabot G, et al. Cyclic administration of pamidronate in children with severe Osteogeneis Imperfecta. N Engl J Med 1998;339(14):986-7.

5. Eiser C, Morse R. A review of measures of quality of life for children with chronic illness. Arch Dis Child 2001;84:205-11.

6. Roizen M, Rodríguez S, Bauer G, Medin G, et al .Initial validation of the Argentinean Spanish version of the PedsQL4.0Generic CoreScales in children and adolescents with chronic diseases: acceptability and comprehensibility in low-income settings. Health Qual Life Outcomes 2008 Aug7; 6:59.

7. Roizen M, Figueroa C,Salvia L.Calidad de vida relacionada con la salud en niños con enfermedades crónicas: comparación de la visión de los niños, sus padres y sus médicos. Arch Argent Pediatr 2007;105(4):305-13.

8. Widmann RF, Laplaza FJ, Bitan FD, Brooks CE, et al. Quality of life in osteogenesis imperfecta. Int Orthop 2002;26(1):3-6.

9. Kok DH, Sakkers RJ, Janse AJ, Pruijs HE, et al . Quality of life in children with osteogenesis imperfecta treated with oral bisphosphonates (Olpadronate): a 2-year randomized placebo-controlled trial. Eur J Pediatr 2007;166(11):1155-61. Epub 2007 Feb 17.

10. Löwing K, Aström E, Oscarsson KA, Söderhäll S, et al. Effect of intravenous pamidronate therapy on everyday activities in children with osteogenesis imperfecta. Acta Paediatr 2007;96(8):1180-3. Epub 2007 Jun 18.

11. Sillence DO, Senn A and Danks DM. Genetic heterogeneity in Osteogenesis imperfecta. J Med Genet 1979;16(2): 101-16.

12. Fano V, Rodríguez Celin M, del Pino M, Buceta S, et al. Osteogenesis imperfecta: evaluación clínica, funcional, y multidisciplinaria de 65 pacientes. An Pediatr (Barc) 2010; 72(5):324-30.

13. Cohen JS, Biesecker BB. Quality of life in rare genetics conditions: A systematic review of the literature. Am J Med Genet A 2010;152(5):1136-56.

14. Engelbert RH, Gulmans VA, Uiterwaal CS, Helders PJ. Osteogenesis imperfecta in childhood: perceived competence in relation to impairment and disability. Arch Phys Med Rehabil 2001;82(7):943-8.

15. Van Brussel M, Takken T, Uiterwaal CS, Pruijs HJ, et al. Physical training in children with osteogenesis imperfecta. J Pediatr 2008;152(1):111-6, 116.e1. Epub 2007 Oct. 